\title{
Níveis críticos para atributos do solo pela distribuição normal reduzida em culturas anuais de subsistência
} \author{
Maria C. M. R. de Souza ${ }^{5}$ \& Celsemy E. Maia ${ }^{6}$

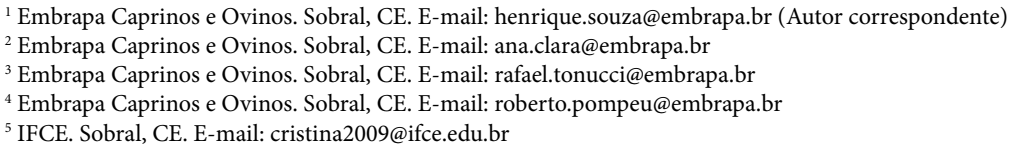

Henrique A. de Souza ${ }^{1}$, Ana C. R. Cavalcante ${ }^{2}$, Rafael G. Tonucci ${ }^{3}$, Roberto C. F. F. Pompeu ${ }^{4}$,

\section{Palavras-chave:}

Zea mays

Vigna unguiculata

fertilidade do solo

semiárido

sertão Inhamuns-Crateús

\begin{abstract}
R E S U M O
Objetivou-se, neste trabalho, propor níveis críticos para atributos químicos do solo utilizando-se a distribuição normal reduzida em áreas de agricultura familiar de subsistência cultivadas com milho e feijão-caupi, no semiárido brasileiro. Amostras de solo foram coletadas em propriedades de agricultores familiares do Sertão dos InhamunsCrateús. Para a obtenção de níveis críticos foi utilizada a distribuição normal reduzida que utiliza os dados de produtividade e a relação entre produtividade e o atributo químico do solo atribuindo o nível crítico para $90 \%$ da produtividade máxima. Os níveis críticos obtidos para as áreas cultivadas com milho e feijão caupi em região semiárida são: $\mathrm{MO}\left(\mathrm{g} \mathrm{kg}^{-1}\right): 7,3$ e 7,5; $\mathrm{pH}: 6,6$ e 6,5; $\mathrm{P}\left(\mathrm{mg} \mathrm{dm}^{-3}\right): 8,6$ e 8,2; $\mathrm{K}: 2,8$ e 2,7; $\mathrm{Ca}\left(\mathrm{mmol}_{\mathrm{c}} \mathrm{dm}^{-3}\right): 33,3$ e 22,4; $\mathrm{Mg}\left(\mathrm{mmol}_{\mathrm{c}} \mathrm{dm}^{-3}\right): 11,2$ e 9,9 e saturação por base (\%): 73 e 71, respectivamente. O nível crítico pela distribuição normal reduzida pode ser utilizado com vista à obtenção de níveis críticos para atributos químicos do solo. Os atributos com maior frequência para o índice negativo em relação ao nível crítico estimado (NCRIz) são Ca $>\mathrm{V}$ $>\mathrm{pH}>\mathrm{Mg}>\mathrm{K}=\mathrm{MO}>\mathrm{Pe} \mathrm{V}>\mathrm{pH}>\mathrm{P}>\mathrm{MO}>\mathrm{Mg}>\mathrm{K}>\mathrm{Ca}$ para as áreas de milho e feijão, respectivamente.
\end{abstract}

Key words:

Zea mays

Vigna unguiculata

soil fertility

semiarid

sertão Inhamuns-Crateús

\section{Critical levels for soil attributes by reduced normal distribution in annual crops of subsistence}

\begin{abstract}
A B S T R A C T
This study aimed to propose critical levels for soil chemical properties using the reduced normal distribution in areas of family subsistence farming under maize and cowpea crops in the Brazilian semiarid. Soil samples were collected on family farms in the region of Inhamuns-Cratéus. To obtain the critical levels the reduced normal distribution was used which uses the productivity data and the relationship between productivity and soil chemical attributes and assigning the critical level for $90 \%$ of the maximum. Critical levels obtained for areas cultivated with maize and cowpea in the semiarid region are: $\mathrm{OM}\left(\mathrm{g} \mathrm{kg}^{-1}\right): 7.3$ and 7.5; $\mathrm{pH}: 6.6$ and $6.5 ; \mathrm{P}\left(\mathrm{mg} \mathrm{dm}^{-3}\right): 8.6$ and $8.2 ; \mathrm{K}\left(\mathrm{mmol}_{c}\right.$ $\left.\mathrm{dm}^{-3}\right): 2.8$ and 2.7; $\mathrm{Ca}\left(\mathrm{mmol} \mathrm{dm}_{c}^{-3}\right): 33.3$ and 22.4; $\mathrm{Mg}\left(\mathrm{mmol} \mathrm{dm}_{c}^{-3}\right): 11.2$ and 9.9; and base saturation (\%): 73 and 71, respectively. The critical level for reduced normal distribution can be used to obtain critical levels for soil chemical properties. The attributes most frequently for negative index over estimated critical level (NCRIz) are $\mathrm{Ca}>\mathrm{V}>\mathrm{pH}>\mathrm{Mg}>\mathrm{K}=\mathrm{OM}>\mathrm{P}$ and $\mathrm{V}>\mathrm{pH}>\mathrm{P}>\mathrm{OM}>\mathrm{Mg}>\mathrm{K}>\mathrm{Ca}$ areas for corn and cowpea, respectively.
\end{abstract}

\section{INTRODUÇÃo}

O manejo do solo exerce influência direta sobre a produtividade das culturas agrícolas sendo determinante seu conhecimento para obtenção de produções economicamente viáveis.

As culturas, e mesmo as cultivares, variam muito na sua capacidade de tolerância ou sensibilidade à acidez ativa, à acidez trocável, saturação por bases, saturação por alumínio e disponibilidade de nutrientes; desta forma, as classes de fertilidade devem ser interpretadas considerando-se as exigências específicas a cada empreendimento agrícola (Alvarez
V. et al., 1999). Segundo Menezes et al. (2012a) os solos da região semiárida apresentam baixa fertilidade natural, alta variabilidade ambiental e pouca informação disponível sobre tecnologias adaptadas aos ecossistemas da região. Ainda os principais processos de perda de nutrientes e água são queimadas, erosão do solo, escoamento e colheita de cultivos e produtos animais (Menezes et al., 2012b).

Conforme Menezes \& Sampaio (2000), a agricultura praticada nas encostas e topos do cristalino na região semiárida inclui o milho e o feijão como os principais produtos e produzem, na maior parte do ano, abaixo das médias regionais 
(800 e $390 \mathrm{~kg} \mathrm{ha}^{-1}$, respectivamente). No semiárido o cultivo do milho e do feijão-caupi é realizado em consórcio principalmente pelos agricultores familiares que procuram obter, de uma mesma área, vários produtos.

Sampaio et al. (1995) citam que baixas doses de fertilizantes nos cultivos no Semiárido produzem retorno econômico e que o não uso de adubos se justifica pelos agricultores acreditarem não compensar o investimento em função da suscetibilidade do clima, especialmente no que diz respeito à irregularidade de chuvas na região.

Considerando que o manejo do solo auxilia na obtenção de boas produções é importante o acompanhamento da fertilidade para o uso correto de adubos e corretivos; entretanto, há poucos trabalhos que auxiliem na interpretação dos resultados da análise de solo principalmente para as culturas do milho e do feijão de subsistência cultivadas sobretudo em região semiárida. Em muitos casos os resultados são elucidados com o uso de níveis críticos e faixas de suficiência provenientes de outras regiões, as quais apresentam características distintas do referido território.

Maia et al. (2001) propuseram a interpretação do diagnóstico nutricional em folhas de café pelo critério da distribuição normal reduzida em que o nível crítico é obtido para cada nutriente considerando-se $90 \%$ da produtividade máxima. Portanto, índices críticos podem colaborar para a orientação na reposição de nutrientes e manejo adequado do solo sobremaneira na agricultura familiar de subsistência.

Diante deste contexto objetiva-se propor níveis críticos para atributos químicos do solo através da distribuição normal reduzida em áreas de agricultura familiar de subsistência, cultivadas com milho e feijão-caupi no semiárido brasileiro.

\section{Material e Métodos}

O trabalho foi realizado com a obtenção de resultado de análise química de solo e produtividade de milho e feijão-caupi cultivados na região do Sertão dos Inhamuns-Cratéus no estado do Ceará, safra 2011/2012. As coletas de solo foram realizadas nas seguintes cidades: Catunda, Independência, Monsenhor Tabosa, Nova Russas, Santa Quitéria e Tamboril. Os principais tipos de solo predominantes nesses municípios são Luvissolos, Argissolos, Planossolos e Neossolos; ainda segundo Lima et al. (2002) a região compreende graus de suscetibilidade a processos erosivos que variam de moderada a muito forte.

As amostras compreendem os resultados de análise de solo realizados em propriedades de agricultores familiares cujo manejo de solo é baseado em broca/destoca da área (retirada de tocos), enleiramento dos garranchos e não utilização do fogo. Outro manejo executado é o cultivo intensivo, sem pousio ou técnicas de conservação e abertura de áreas de capoeira. Entre os manejos que visam à reposição de nutrientes há aplicação de estercos, quando disponíveis. As sementes de milho e feijão utilizadas são dos próprios agricultores (sementes crioulas), armazenadas e conservadas por eles cujas estratégias de conservação de sementes podem ser verificadas em Catão et al. (2010) e Vasconcelos \& Mata (2011); há, também, agricultores que utilizam sementes provenientes do governo estadual (Empresa de Assistência Técnica e Extensão Rural do Ceará).

O plantio é realizado em covas e em consórcio, com média de cinco sementes de milho e três de feijão; o espaçamento entre linhas é variável $(\sim 1,0 \times 0,5 \mathrm{~m})$ em função do número de fileiras de feijão ( 2 a 3 ) entre as plantas de milho, Morgado \& Willey (2008) relatam o consórcio de milho e feijão em região semiárida; não há uso de fertilizantes sintéticos.

Os teores médios de argila, silte, areia fina e areia grossa foram de 9, 20, 28 e 43\%, respectivamente. Os valores médios históricos de precipitação para a região do Sertão dos InhamunsCrateús são de $707 \mathrm{~mm}$ anuais.

Trabalhou-se com 46 e 42 amostras de solo de áreas cultivadas com milho e feijão caupi, respectivamente, das quais 36 amostras são de áreas com consórcio entre milho e feijão.

As amostras de solo foram coletadas no início do período chuvoso, entre os meses de dezembro de 2011 a abril de 2012. Os atributos químicos avaliados foram matéria orgânica (MO), $\mathrm{pH}$ (água), fósforo $(\mathrm{P})$, potássio $(\mathrm{K})$, cálcio $(\mathrm{Ca})$, magnésio $(\mathrm{Mg})$ e saturação por bases $(\mathrm{V})$, sendo que as variáveis foram determinadas segundo EMBRAPA (1997), exceto a V, que foi calculada $(\mathrm{V}=$ (soma de bases/capacidade de troca catiônica $\left.{ }^{\star} 100\right)$.

Para obtenção do nível crítico no solo pela distribuição contínua de probabilidade segundo Maia et al. (2001), utilizaram-se os dados de produtividade $(\mathrm{P})$ e de $\mathrm{Q}$, em que $\mathrm{Q}$ é definido como a relação entre $\mathrm{P}$ e $\mathrm{n}_{\mathrm{i}}\left(\mathrm{Q}=\mathrm{P} / \mathrm{n}_{\mathrm{i}}\right)$, sendo $\mathrm{n}_{\mathrm{i}} \mathrm{o}$ atributo do solo para o qual se deseja encontrar o nível crítico que determina o valor da produtividade que representa $90 \%$ do seu valor máximo. Para o milho, o nível crítico no solo foi calculado pela Eq. 1 para pH, Ca e V e pela Eq. 2 para MO, $\mathrm{P}, \mathrm{K}, \mathrm{Mg}$; já para o feijão o nível crítico foi calculado pela Eq. 3 para M.O, pH, P, K, Mg e V e para o Ca, pela Eq. 4. Na Eq. 1 os valores de P e Q apresentam normalidade, na Eq. 2 os valores de $\mathrm{P}$ apresentam normalidade e os dados de $\mathrm{Q}$ foram transformados em raiz quadrática, na Eq. 3 os valores de $\mathrm{P}$ e $\mathrm{Q}$ foram transformados em raiz quadrática e na Eq. 4 os valores de $\mathrm{P}$ foram transformados em raiz quadrática e os dados de $\mathrm{Q}$ foram transformados em logaritmo natural.

$$
\begin{gathered}
\mathrm{NC}_{\mathrm{i}}=\frac{1,281552 \mathrm{~s}_{1}+\mathrm{m}_{1}}{1,281552 \mathrm{~s}_{2}+\mathrm{m}_{2}} \\
\mathrm{NC}_{\mathrm{i}}=\frac{1,281552 \mathrm{~s}_{1}+\mathrm{m}_{1}}{\left(1,281552 \mathrm{~s}_{2}+\mathrm{m}_{2}\right)^{2}} \\
\mathrm{NC}_{\mathrm{i}}=\frac{\left(1,281552 \mathrm{~s}_{1}+\mathrm{m}_{1}\right)^{2}}{\left(1,281552 \mathrm{~s}_{2}+\mathrm{m}_{2}\right)^{2}} \\
\mathrm{NC}_{\mathrm{i}}=\frac{\left(1,281552 \mathrm{~s}_{1}+\mathrm{m}_{1}\right)^{2}}{\mathrm{e}^{\left(1,281552 \mathrm{~s}_{2}+\mathrm{m}_{2}\right)}}
\end{gathered}
$$

em que:

$\mathrm{m}_{1}$ e $\mathrm{s}_{1}$ - média aritmética e o desvio-padrão de $\mathrm{P}$ 
$\mathrm{m}_{2}$ e $\mathrm{s}_{2}$ - média e o desvio-padrão de $\mathrm{Q}$, respectivamente

A pressuposição básica para se encontrar o nível crítico pela distribuição contínua de probabilidade é a de que os dados de $\mathrm{Pe}$ Q sigam distribuição normal; para tanto, testou-se a normalidade das variáveis citadas empregando-se o teste de Shapiro Wilk ( $\mathrm{p}$ $>0,05)$. Quando não se observou a normalidade os dados foram transformados por raiz quadrada ou logaritmo natural.

Ainda de posse dos níveis críticos obtidos pela distribuição normal reduzida para cada atributo de solo $(\mathrm{pH}, \mathrm{MO}, \mathrm{P}, \mathrm{Ca}, \mathrm{Mg}$ e V) calculou-se o índice para verificar se os resultados obtidos nas análises de solos dessas variáveis estariam limitando a produtividade dessas áreas, pela Eq. 5

$$
\mathrm{I}_{\mathrm{i}}=\frac{\text { Asolo }}{\mathrm{NCRIz}_{\mathrm{i}}}-1
$$

sendo:

i - característica avaliada

Asolo - resultado da análise do solo

$\mathrm{NCRIz}_{\mathrm{i}}$ - nível crítico estimado. Quanto mais negativo $\mathrm{I}_{\mathrm{i}}$, mais deficiente estará o solo; assim, os valores negativos foram somados e apresentada a frequência de cada atributo deficiente em relação a NCRIz estimada

Utilizou-se o programa estatístico SISVAR (Ferreira, 2011) para realizar o teste de normalidade.

\section{Resultados e Discussão}

Na Tabela 1 é apresentada a estatística descritiva dos dados de análise de fertilidade do solo para as áreas de milho e feijãocaupi.

Entre os resultados obtidos para a fertilidade do solo observam-se maiores variações (CV) em ordem decrescente em $\mathrm{K}>\mathrm{P}>\mathrm{Mg}>\mathrm{MO}>\mathrm{Ca}>\mathrm{V}>\mathrm{pH}$ e em $\mathrm{K}>\mathrm{P}>\mathrm{Ca}>\mathrm{Mg}>$ $\mathrm{MO}>\mathrm{V}>\mathrm{pH}$, para milho e feijão, respectivamente. Os altos valores de CV são justificáveis em função da diversidade de solos, precipitação e manejos que são empregados nas culturas avaliadas (pousio, adubo orgânico, destoca/broca, ...). Rozane

Tabela 1. Valores médios, mínimo e máximo, desvio padrão e coeficiente de variação (CV) das amostras de solo nas áreas cultivadas com milho e feijão

\begin{tabular}{|c|c|c|c|c|c|c|c|c|}
\hline & \multirow{2}{*}{$\begin{array}{c}\text { Produção } \\
\text { kg ha-1 }^{-1}\end{array}$} & \multirow[b]{2}{*}{$\mathrm{pH}$} & \multirow{2}{*}{$\begin{array}{c}\mathrm{MO} \\
\mathrm{g} \mathrm{kg}^{-1}\end{array}$} & \multirow{2}{*}{$\begin{array}{c}P \\
\mathrm{mg} \mathrm{kg}^{-1}\end{array}$} & K & $\mathrm{Ca}$ & $\mathrm{Mg}$ & V \\
\hline & & & & & \multicolumn{3}{|c|}{$\mathrm{mmol}_{\mathrm{c}} \mathrm{dm}^{-3}$} & $\%$ \\
\hline & \multicolumn{8}{|c|}{ Milho $(n=46)$} \\
\hline Média & 432 & 6,6 & 9,8 & 29,4 & 5,8 & 30,8 & 15,7 & 71,5 \\
\hline Mínimo & 100 & 5,2 & 3,3 & 3,0 & 1,1 & 13,0 & 5,0 & 34,0 \\
\hline Máximo & 900 & 7,8 & 16,7 & 151,0 & 59,0 & 53,0 & 36,0 & 98,0 \\
\hline Desv & 198 & 0,5 & 3,5 & 35,1 & 9,4 & 10,5 & 8,1 & 13,8 \\
\hline \multirow[t]{2}{*}{ CV (\%) } & 45,7 & 7,3 & 35,4 & 119,4 & 162,6 & 34,2 & 51,9 & 19,4 \\
\hline & \multicolumn{8}{|c|}{ Feijão $(n=42)$} \\
\hline Média & 330 & 6,6 & 9,8 & 22,6 & 5,6 & 34,9 & 15,3 & 71,3 \\
\hline Mínimo & 60 & 5,2 & 3,2 & 3,0 & 1,1 & 8,0 & 5,0 & 34,0 \\
\hline Máximo & 750 & 7,8 & 16,7 & 123,0 & 59,0 & 84,0 & 36,0 & 98,0 \\
\hline Desvio padrão & 170 & 0,5 & 3,7 & 25,4 & 8,9 & 19,7 & 8,6 & 13,8 \\
\hline CV (\%) & 51,4 & 7,9 & 37,2 & 112,6 & 158,0 & 56,5 & 56,3 & 19,3 \\
\hline
\end{tabular}

et al. (2011) obtiveram, em dimensionamento do número de amostras para avaliação da fertilidade do solo, as maiores variações nos seguintes atributos químicos do solo em ordem decrescente $\mathrm{Ca}>\mathrm{Mg}>\mathrm{K}>\mathrm{P}>\mathrm{V}>\mathrm{MO}>\mathrm{pH}$ e $\mathrm{K}>\mathrm{P}>\mathrm{Ca}>$ $\mathrm{Mg}>\mathrm{MO}>\mathrm{V}>\mathrm{pH}$ para milho e pastagem, respectivamente.

Após a aplicação do teste de normalidade e obtenção de resultados não significativos houve necessidade de transformação em raiz quadrada para $\mathrm{MO}, \mathrm{P}, \mathrm{K}$ e $\mathrm{Mg}$ nas avaliações em referência às áreas cultivadas com o milho. Os valores de média e desvio-padrão de cada atributo químico do solo e seu respectivo nível crítico e literatura de referência estão na Tabela 2 para áreas cultivadas com milho.

Tabela 2. Valores da média e desvio padrão da relação entre produtividade e atributos químicos do solo e nível crítico em áreas $(\mathrm{n}=46)$ cultivadas com milho obtidas pelo método da distribuição contínua de probabilidade (NCRiz)

\begin{tabular}{|c|c|c|c|c|}
\hline Relação & Média & $\begin{array}{l}\text { Desvio } \\
\text { padrão }\end{array}$ & NCRIz & $\begin{array}{l}\text { Nível crítico } \\
(\text { (UFC, 1993) }\end{array}$ \\
\hline Produtividade (Prod.) & $\begin{array}{c}432 \\
\left(\mathrm{~kg} \mathrm{ha}^{-1}\right)\end{array}$ & $\begin{array}{c}197 \\
\left(\mathrm{~kg} \mathrm{ha}^{-1}\right)\end{array}$ & - & - \\
\hline Prod./M0 1 & $6,7^{\star}$ & $2,2^{*}$ & $\begin{array}{c}7,3 \\
\left(\mathrm{~g} \mathrm{kq}^{-1}\right)\end{array}$ & $\begin{array}{c}15,0 \\
\left(\mathrm{~g} \mathrm{~kg}^{-1}\right)\end{array}$ \\
\hline Prod./pH & $65,7^{*}$ & $29,7^{*}$ & 6,6 & 6,5 \\
\hline Prod./P1 & $5,6^{*}$ & $2,6^{*}$ & $\begin{array}{c}8,6 \\
\left(\mathrm{mg} \mathrm{dm}^{-3}\right)\end{array}$ & $\begin{array}{c}10,0 \\
\left(\mathrm{mg} \mathrm{dm}^{-3}\right)\end{array}$ \\
\hline Prod./K $\mathrm{K}^{1}$ & $10,7^{*}$ & $3,9^{*}$ & $\begin{array}{c}2,8 \\
\left(\mathrm{mmol}_{\mathrm{c}} \mathrm{dm}^{-3}\right)\end{array}$ & $\begin{array}{c}1,2 \\
\left(\mathrm{mmol}_{\mathrm{c}} \mathrm{dm}^{-3}\right)\end{array}$ \\
\hline Prod./Ca & $13,5^{*}$ & $6,4^{*}$ & $\begin{array}{c}33,3 \\
\left(\mathrm{mmol}_{\mathrm{c}} \mathrm{dm}^{-3}\right)\end{array}$ & $\begin{array}{c}15,0 \\
\left(\mathrm{mmol}_{\mathrm{c}} \mathrm{dm}^{-3}\right)\end{array}$ \\
\hline Prod./Mg ${ }^{1}$ & $5,4^{*}$ & $1,8^{*}$ & $\begin{array}{c}11,2 \\
\left(\mathrm{mmol}_{\mathrm{c}} \mathrm{dm}^{-3}\right)\end{array}$ & $\begin{array}{c}5,0 \\
\left(\mathrm{mmol}_{\mathrm{c}} \mathrm{dm}^{-3}\right)\end{array}$ \\
\hline Prod./V & $6,0^{*}$ & $2,6^{*}$ & $\begin{array}{l}73,0 \\
(\%)\end{array}$ & - \\
\hline \multicolumn{5}{|c|}{ Produtividade para $90 \%$ da máxima: $685 \mathrm{~kg} \mathrm{ha}^{-1}$} \\
\hline
\end{tabular}

${ }^{1}$ As médias e desvio padrão foram transformados em raiz quadrada

${ }^{2}$ Recomendações de Adubação e Calagem para o Estado do Ceará - UFC (1993). Valores provenientes da faixa superior da classificação baixa

${ }^{\star}$ Valor da relação entre a produtividade e 0 atributo do solo

Com relação à literatura padrão (UFC, 1993) foram considerados níveis críticos os valores superiores da faixa de interpretação baixa verificando-se, então, que os valores de $\mathrm{pH}$ e P estão próximos aos sugeridos para o estado do Ceará porém para K, Ca e Mg os níveis obtidos são superiores à UFC (1993).

Tais resultados apontam a necessidade de maior reposição de nutrientes (cátions básicos principalmente) ou correção do solo para obtenção de produções regulares em função dos padrões (NC) obtidos, ou seja, essas áreas apresentarão produções maiores quando as concentrações no solo forem próximas ou superiores ao NCRIz calculado.

Em levantamento realizado por Menezes et al. (2012b) os valores médios de carbono orgânico do bioma Caatinga estão em torno de $9,3 \mathrm{~g} \mathrm{~kg}^{-1}$, considerando-se que $58 \%$ da matéria orgânica é carbono, o valor médio seria de $15,9 \mathrm{~g} \mathrm{~kg}^{-1}(\mathrm{MO})$; assim, o resultado apresentado na Tabela 2 é aquém do sugerido pela literatura referência e Menezes et al. (2012b), o que é justificável em função da não reposição de nutrientes (baixo uso de estercos na área) e manejos pouco conservacionistas 
empregados por alguns agricultores como cultivo intensivo, não adoção do pousio e abertura de áreas de capoeira.

Na Tabela 3 estão apresentados a média, o desvio padrão, o nível crítico e os índices da literatura de referência para as áreas que foram cultivadas com feijão caupi. Após a aplicação do teste de normalidade foi constatada a necessidade de transformação dos dados de produtividade e dos atributos químicos do solo em raiz quadrada, exceto para cálcio cujos dados foram transformados para logaritmo natural.

Tabela 3. Valores da média e desvio padrão da relação entre produtividade e atributos químicos do solo e nível crítico em áreas $(n=42)$ cultivadas com feijão obtidos pelo método da distribuição contínua de probabilidade (NCRiz)

\begin{tabular}{|c|c|c|c|c|}
\hline Relação & Média & $\begin{array}{l}\text { Desvio } \\
\text { padrão }\end{array}$ & NCRIz & $\begin{array}{l}\text { Nível crítico } \\
\text { (UFC, 1993) }^{3}\end{array}$ \\
\hline Produtividade (Prod.) ${ }^{1}$ & $\begin{array}{c}330 \\
\left(\mathrm{~kg} \mathrm{ha}^{-1}\right)\end{array}$ & $\begin{array}{c}170 \\
\left(\mathrm{~kg} \mathrm{ha}^{-1}\right)\end{array}$ & - & ( \\
\hline Prod./M0 ${ }^{1}$ & $6,0^{*}$ & $2,1^{*}$ & $\begin{array}{c}7,5 \\
\left(\mathrm{~g} \mathrm{~kg}^{-1}\right)\end{array}$ & $\begin{array}{c}15,0 \\
\left(\mathrm{~g} \mathrm{~kg}^{-1}\right)\end{array}$ \\
\hline Prod./pH ${ }^{1}$ & $6,9^{*}$ & $1,9^{*}$ & 6,5 & 6,5 \\
\hline Prod./P $P^{1}$ & $5,1^{*}$ & $2,6^{*}$ & $\begin{array}{c}8,2 \\
\left(\mathrm{mg} \mathrm{dm}^{-3}\right)\end{array}$ & $\begin{array}{c}10,0 \\
\left(\mathrm{mg} \mathrm{dm}^{-3}\right)\end{array}$ \\
\hline Prod./K $\mathrm{K}^{1}$ & $9,4^{\star}$ & $3,9^{*}$ & $\begin{array}{c}2,7 \\
\left(\mathrm{mmol}_{\mathrm{c}} \mathrm{dm}^{-3}\right)\end{array}$ & $\left(\mathrm{mmol}_{\mathrm{c}} \mathrm{dm}^{-3}\right)$ \\
\hline Prod./Ca² & $2,3^{*}$ & $0,7^{*}$ & $\begin{array}{c}22,4 \\
\left(\mathrm{mmol}_{\mathrm{c}} \mathrm{dm}^{-3}\right)\end{array}$ & $\begin{array}{c}15,0 \\
\left(\mathrm{mmol}_{\mathrm{c}} \mathrm{dm}^{-3}\right)\end{array}$ \\
\hline Prod./Mg ${ }^{1}$ & $5,2^{*}$ & $2,1^{*}$ & $\begin{array}{c}9,9 \\
\left(\mathrm{mmol}_{\mathrm{c}} \mathrm{dm}^{-3}\right)\end{array}$ & $\begin{array}{c}5,0 \\
\left(\mathrm{mmol}_{\mathrm{c}} \mathrm{dm}^{-3}\right)\end{array}$ \\
\hline Prod./N ${ }^{1}$ & $2,1^{*}$ & $0,6^{*}$ & $\begin{array}{c}71,0 \\
(\%)\end{array}$ & - \\
\hline \multicolumn{5}{|c|}{ Produtividade para $90 \%$ da máxima: $548 \mathrm{~kg} \mathrm{ha}^{-1}$} \\
\hline
\end{tabular}

${ }^{1}$ As médias e desvio padrão foram transformados em raiz quadrada

${ }^{2}$ As médias e desvio padrão foram transformados em logaritmo natural

${ }^{3}$ Recomendações de Adubação e Calagem para o Estado do Ceará - UFC (1993). Valores provenientes da faixa superior da classificação baixa

* Valor da relação entre a produtividade e 0 atributo do solo

Comparando os valores obtidos entre o nível crítico e a literatura de referência (Tabela 3 ) pode-se realizar análise análoga à apresentada para os dados da Tabela 2, ou seja, valores próximos para $\mathrm{pH}$ e $\mathrm{P}$, inferiores para $\mathrm{MO}$ e superiores para os cátions básicos em relação aos preconizados por UFC (1993).

Em trabalho no qual foram avaliadas as concentrações de P (fósforo) em classes de solos da Paraíba e Pernambuco, Silveira et al. (2006), constataram que os resultados das extrações de Mehlich-1 em 69 amostras de solo mostram uma variabilidade tão grande quanto a encontrada para o $\mathrm{P}$ total e ainda maior dentro de cada ordem. Os teores de $\mathrm{P}$ variaram de 1 a $202 \mathrm{mg} \mathrm{kg}^{-1}$.

Os limites de $\mathrm{P}$ de 20 e $10 \mathrm{mg} \mathrm{kg}^{-1}$ têm sido considerados como os de disponibilidade alta e média, respectivamente (Silveira et al., 2006).

Os valores obtidos para $P$ nas Tabelas 2 e 3 estão condizentes, portanto, com as concentrações observadas em outras avaliações. Especificamente para essas situações considera-se que o uso de insumos como adubos sintéticos não é prática rotineira para a agricultura familiar de subsistência.

De forma geral o teor de $\mathrm{P}$ disponível para as plantas é baixo na maior parte da região semiárida. Neste contexto, a ciclagem de fósforo está relacionada principalmente ao $\mathrm{P}$ nativo do solo. Estratégias como o uso de resíduos orgânicos, quando disponível devem ser adotadas (Silva et al., 1999; Sampaio et al., 2004; Moreira et al., 2006; Salcedo, 2006).

Alves et al. (2010), verificaram que o nível crítico de potássio (K) em solos da Zona da Mata de Pernambuco variou de 49 a $109 \mathrm{mg} \mathrm{dm}^{-3}$ em solos cultivados com nem; o nível crítico para

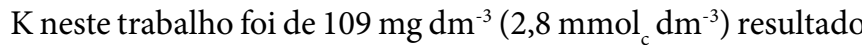
dentro da faixa obtida pelos referidos autores.

$\mathrm{Na}$ Tabela 4 é apresentada a frequência de resultados com índices negativos em relação a NCRIz calculada para as áreas de milho e feijão.

Tabela 4. Frequência de valores com índice negativo em relação a NCRIz para atributos químicos do solo em áreas cultivadas com milho e feijão

\begin{tabular}{lccccccc}
\hline & $\mathbf{p H}$ & $\mathbf{M O}$ & $\mathbf{P}$ & $\mathbf{K}$ & $\mathbf{C a}$ & $\mathbf{M g}$ & $\mathbf{V}$ \\
\cline { 2 - 7 } & & & & $\%$ & & & \\
Milho & 43,5 & 27,0 & 24,3 & 27,0 & 59,1 & 34,4 & 50,0 \\
Feijão & 35,7 & 31,0 & 31,4 & 28,6 & 14,3 & 29,2 & 45,2 \\
\hline
\end{tabular}

Os atributos que apresentaram valor decrescente em relação ao índice para as áreas de milho e feijão foram $\mathrm{Ca}>\mathrm{V}$ $>\mathrm{pH}>\mathrm{Mg}>\mathrm{K}=\mathrm{MO}>\mathrm{Pe} \mathrm{V}>\mathrm{pH}>\mathrm{P}>\mathrm{MO}>\mathrm{Mg}>\mathrm{K}>\mathrm{Ca}$, respectivamente.

Para saturação por bases o nível obtido se relaciona ao valor pH. Prado (2001) sugere elevação da saturação por bases para $65 \%$ na cultura do milho; na cultura do feijão Fonseca et al. (2010) citam 60\%; no entanto, no presente estudo as espécies utilizadas são crioulas ou variedades (provenientes dos programas governamentais), indicando valores maiores para saturação por bases, resultado que é corroborado pelas frequências apresentadas na Tabela 4.

Segundo Alves \& Amaral (2011) e Lima (2012), a produção média de milho e feijão caupi no estado do Ceará foi de 1.313 e $440 \mathrm{~kg} \mathrm{ha}^{-1}$ na safra 2010/2011, respectivamente mas para o nível crítico proposto o valor de $685 \mathrm{~kg} \mathrm{ha}^{-1}$ para milho estaria abaixo da média e para feijão caupi o valor de $548 \mathrm{~kg} \mathrm{ha}^{-1}$ estaria acima da média do estado.

Há de ressaltar que os resultados foram obtidos em condições de agricultura de subsistência, ou seja, com baixo nível tecnológico. Considerando que as médias dessas culturas foram 432 e $330 \mathrm{~kg} \mathrm{ha}^{-1}$ para milho e feijão (Tabela 1), respectivamente, relacionado ao alto valor do CV (alta variabilidade) indica o incremento da produção que pode ser obtido quando melhorados os atributos químicos do solo constantes nas Tabelas 2 e 3 . A mudança de patamar de produção permite que novos índices sejam utilizados visando às maiores produções para essas culturas.

Alguns manejos preconizados como o uso de esterco e cultivo em aleias nas áreas de agricultura podem ser boa opção para melhoria da fertilidade do solo e consequente aumento da produção em especial para agricultores familiares. $\mathrm{O}$ uso de insumos como adubos minerais é pouco empregado nas culturas agrícolas da região semiárida, seja pelo risco proveniente do clima ou até mesmo por desconhecimento dos efeitos benéficos de sua aplicação (Menezes et al., 2012a); apesar disto, 
o uso desses insumos pode propiciar patamares de produção superiores aos verificados na Tabela 1 .

Há, na literatura, trabalhos que retratam o uso de esterco e ramas de leguminosas como aporte de nutrientes (principalmente para $\mathrm{N}$ e $\mathrm{P}$ ), com resultados positivos na manutenção da fertilidade do solo e obtenção de boas produtividades para milho e feijão (Oliveira, 1993; Oliveira et al., 2001; Perez Marin et al., 2006; Silva \& Menezes, 2007; Menezes \& Salcedo, 2007; Silva et al., 2011; Primo et al., 2012).

Ainda quanto às variações de armazenamento de água no perfil do solo acompanham as variações de precipitação (Antonino et al., 2000), independente do manejo executado (presença de cobertura morta) influenciando também as produtividades obtidas; há de se ressaltar, assim, a estreita relação entre o patamar de produção com as precipitações do período de cultivo e o manejo de solo.

A vantagem do referido método trabalhado (NCRiz) reside na proposição de nível de suficiência considerando-se os manejos locais (uso de estercos, leguminosas, abertura de áreas, destoca, broca etc.). Camacho et al. (2012) citam a possibilidade de uso do NCRiz para obtenção de níveis críticos em regiões específicas sem a necessidade de ensaios regionais; portanto, os dados apresentados podem ser uma primeira aproximação de níveis críticos de atributos químicos do solo em áreas cultivadas para subsistência no semiárido brasileiro.

\section{Conclusões}

1. O nível crítico pela distribuição normal reduzida pode ser utilizado para obtenção de níveis críticos para atributos químicos do solo.

2. Os níveis críticos obtidos para as áreas cultivadas cuja análise foi realizada em áreas cultivadas com milho e feijão caupi em região semiárida, são: $\mathrm{MO}\left(\mathrm{g} \mathrm{kg}^{-1}\right)$ : 7,3 e 7,5; pH: 6,6 e 6,5; P (mg dm $\left.{ }^{-3}\right): 8,6$ e 8,2; K: 2,8 e 2,7; $\mathrm{Ca}\left(\mathrm{mmol}_{\mathrm{c}} \mathrm{dm}^{-3}\right): 33,3$ e 22,4; $\mathrm{Mg}\left(\mathrm{mmol} \mathrm{dm}^{-3}\right): 11,2$ e 9,9 e saturação por base (\%): 73 e 71 , respectivamente.

3. Os atributos com maior frequência para o índice negativo em relação ao NCRIz são $\mathrm{Ca}>\mathrm{V}>\mathrm{pH}>\mathrm{Mg}>\mathrm{K}=\mathrm{MO}>\mathrm{P}$ e V $>\mathrm{pH}>\mathrm{P}>\mathrm{MO}>\mathrm{Mg}>\mathrm{K}>\mathrm{Ca}$ para as áreas de milho e feijão, respectivamente.

\section{Agradecimentos}

Ao IDEF (Instituto de Desenvolvimento da Economia Familiar) pelo auxílio na execução do trabalho, coleta de solo e informações com os agricultores assistidos pelo Projeto Suporte Forrageiro.

\section{Literatura Citada}

Alvarez V., V. H.; Novais, R. F.; Barros, N. F.; Cantarutti, R. B.; Lopes, A. S. Interpretação dos resultados das análises de solos. In: Ribeiro, A. C.; Guimarães, P. T. G.; Alvarez V., V. H. Recomendações para uso de corretivos e fertilizantes em Minas Gerais - 5 Aproximação. Viçosa: Comissão de Fertilidade do Solo do Estado de Minas Gerais, 1999. p.25-32.
Alves, A. R.; Passos, M. A. A.; Holanda, A. C. Níveis críticos de potássio para o crescimento de nim (Azdirachta indica A. juss.) em solos da zona da mata de Pernambuco. Revista Verde de Agroecologia e Desenvolvimento Sustentável, v.5, p.58-71, 2010.

Alves, H. C. R.; Amaral, R. F. Produção, área colhida e produtividade do milho no nordeste. Fortaleza: Banco do Nordeste, 2011. 9p. Informe Rural Etene, 16

Antonino, A. C. D.; Sampaio, E. V. S. B.; Dallolio, A.; Salcedo, I. H. Balanço hídrco em solo com cultivos de subsistência no semi-árido do nordeste do Brasil. Revista Brasileira de Engenharia Agrícola e Ambiental, v.4, p.29-34, 2000.

Camacho, M. A.; Silveira, M. V.; Camargo, R. A.; Natale, W. Faixas normais de nutrientes pelos métodos ChM, DRIS e CND e nível crítico pela método de distribuição normal reduzida para laranjeira-pera. Revista Brasileira de Ciência do Solo, v.36, p.193-200, 2012.

Catão, H. C. R. M.; Costa, F. M.; Valadares, S. V.; Dourado, E. R.; Brandão Júnior, D. S.; Sales, N. L. P. Qualidade física, fisiológica e sanitária de sementes de milho crioulo produzidas no norte de Minas Gerais. Ciência Rural, v.40, p.2060-2066, 2010.

EMBRAPA - Empresa Brasileira de Pesquisa Agropecuária. Manual de métodos de análise de solo. Rio de Janeiro: Embrapa CNPS, 1997. 212p. Documentos; 1

Ferreira, D. F. SISVAR: A computer statistical analysis system. Ciência e Agrotecnologia, v.35, p.1039-1042, 2011.

Fonseca, M. R.; Fernandes, A. R.; Silva, G. R.; Brasil, E. C. Teor e acúmulo de nutrientes por plantas de feijão caupi em função do fósforo e da saturação por bases. Revista de Ciências Agrárias, v.53, p.195-205, 2010.

Lima, A. A. C.; Oliveira, F. N. S.; Aquino, A. R. L. Limitações do uso dos solos do estado do Ceará por suscetibilidade à erosão. Fortaleza: Embrapa Agroindústria Tropical, 2002. 19p. Documentos, 54

Lima, G. A. S. Produção de feijão no Ceará. Fortaleza: CONAB, 2012. 2p. Conjuntura Mensal

Maia, C. E.; Morais, E. R. C.; Oliveira, M. Nível crítico pelo critério da distribuição normal reduzida: uma nova proposta para interpretação foliar. Revista Brasileira de Engenharia Agrícola e Ambiental, v.5, p.235-238, 2001.

Menezes, R. S. C.; Primo, D. C.; Primo, D. C.; Martins, J. C. R.; Jesus, K. N.; Althoff, T. D. Fertilidade dos solos no semi-árido. In: Reunião Brasileira de Fertilidade do Solo e Nutrição de Plantas, 30, Maceió, 2012. Anais...Maceió: SBCS, 2012a. 41p.

Menezes, R. S. C.; Salcedo, I. H. Mineralização de N após a incorporação de adubos org6anicos em um Neossolo Regolítico cultivado com milho. Revista Brasileira de Engenharia Agrícola e Ambiental, v.11, p.361-367, 2007.

Menezes, R. S. C.; Sampaio, E. V. S. B. Agricultura sustentável no semi-árido nordestino. In: Oliveira, T. S.; Assis Júnior, R. N.; Romero, R. E.; Silva, J. R. C. Agricultura, sustentabilidade e o semi-árido. Fortaleza: UFC, Viçosa: SBCS, 2000. p.20-46. 
Menezes, R. S. C.; Sampaio, E. V. S. B.; Giongo, V.; Pérez-Marin, A. M. Biogeochemical cycling in terrestrial ecosystems of the Caatinga Biome. Brazilian Journal of Biology, v.72, p.643-653, 2012b.

Moreira, F. L. M.; Mota, F. O. B.; Clemente, C. A.; Azevedos, B. M.; Bomfim, G. V. Adsorção de fósforo em solos do Estado do Ceará. Revista Ciência Agronômica, v.37, p. 7-12, 2006.

Morgado, L. B.; Willey, R. W. Optimum plant population ofr maize-bean intercropiing system in the Brazilian semi-arid region. Scientia Agricola, v.65, p.474-480, 2008.

Oliveira, A. P.; Araújo, J. S.; Alves, E. U.; Noronha, M. A. S.; Cassimiro, C. M.; Mendonça, F. G. Rendimento de feijãocaupi cultivado com esterco bovino e adubo mineral. Horticultura Brasileira, v.19, p.81-84, 2001.

Oliveira, F. J. Combinações de espaçamento e populações de plantas de caupi e de milho em monocultura e consorciados. Pesquisa Agropecuária Brasileira, v.28, p.931-945, 1993.

Pérez Marin, A. M.; Menezes, R. S. C.; Silva, E. D.; Sampaio, E. V. S. B. Efeito da Gliricídia sepium sobre nutrientes do solo, microclima e produtividade do milho em sistema agroflorestal no agreste paraibano. Revista Brasileira de Ciência do Solo, v.30, p.555-564, 2006.

Prado, R. M. Saturação por bases e híbridos de milho sob sistema de plantio direto. Scientia Agrícola, v.58, p.391-394, 2001.

Primo, D. C.; Menezes, R. S. C.; Silva, T. O.; Garrido, M. S.; Cabral, P. K. T. Contribuição da adubação orgânica na absorção de nutrientes e na produtividade de milho no semiárido paraibano. Revista Brasileira de Ciências Agrárias, v.7, p.81-88, 2012.

Rozane, D. E.; Romualdo, L. M.; Centurion, J. F.; Barbosa, J. C. Dimensionamento do número de amostras para avaliação da fertilidade do solo. Semina:Ciências Agrárias, v.32, p.111-118, 2011.

Salcedo, I. H. Biogeoquímica do fósforo em solos da região Semi-árida do NE do Brasil. Revista Geografia, v.23, p.159184, 2006.
Sampaio, E. V. S. B.; Salcedo, I. H.; Silva, F. B. R. Fertilidade de Solos do Semi-Árido do Nordeste. In: Pereira, J. R.; Faria, C. M. B. Fertilizantes: insumo básico para agricultura e combate à fome. Petrolina: Embrapa SBCS, 1995. p.51-71.

Sampaio, E. V. S. B.; Tiessen, H.; Antonino, A. C. D.; Salcedo, I. $\mathrm{H}$. Residual $\mathrm{N}$ and $\mathrm{P}$ fertilizer effect and fertilizer recovery on intercropped and sole-cropped corn and bean in semiarid northeast Brazil. Nutrient Cycling in Agroecosystems, v.70, p.1-11, 2004.

Silva, A. B.; Resende, M. Sousa, A. R.; Margolis, E. Mobilização do solo, erosão e produtividade de milho e feijão em um Regossolo no agreste pernambucano. Pesquisa Agropecuária Brasileira, v.34, p.299-307, 1999.

Silva, T. O.; Menezes, R. S. C. Adubação orgânica da batata com esterco e, ou, Crotalaria juncea. II - disponibilidade de N, P e K no solo ao longo do ciclo de cultivo. Revista Brasileira de Ciência do Solo, v.31, p.51-61, 2007.

Silva, T. O.; Menezes, R. S. C.; Alves, R. N.; Primo, D. C.; Silva, G. B. M. S. Produtividade de grãos e frações nitrogenadas do milho submetido a manejo de adubos orgânicos na região semiárida. Semina: Ciências Agrárias, v.32, p.1735$1744,2011$.

Silveira, M. M. L.; Araújo, M. S. B.; Sampaio, E. V. S. B. Distribuição de fósforo em diferentes ordens de solo do semi-árido da Paraíba e de Pernambuco. Revista Brasileira de Ciência do Solo, v.30, p.281-291, 2006.

UFC - Universidade Federal do Ceará. Recomendações de adubação e calagem para o Estado do Ceará. Fortaleza: Imprensa Universitária, 1993. 247p.

Vasconcelos, J. M. G.; Mata, M. F. Casas de sementes comunitárias: estratégias de sustentabilidade alimentar e preservação da biodiversidade no semi-árido cearense. Cadernos de Agroecologia, v.6, p.1-5, 2011. 